\title{
DETERMINATION OF TIME DEPENDENT DIFFUSION COEFFICIENT IN TIME FRACTIONAL DIFFUSION EQUATIONS BY FRACTIONAL SCALING TRANSFORMATIONS METHOD
}

\author{
MINE AYLIN BAYRAK ${ }^{a}$ AND ALI DEMIR ${ }^{b}$ \\ Department of Mathematics, Kocaeli University, Izmit, Kocaeli, Turkey. \\ ${ }^{a}$ E-mail: aylin@kocaeli.edu.tr \\ ${ }^{b}$ E-mail: ademir@kocaeli.edu.tr \\ \|\|
}

Abstract

This study is devoted to investigation of inverse problem of identifying unknown time-dependent diffusion coefficient in time fractional diffusion equation in the sense of the modified Riemann-Liouville fractional derivative, by employing fractional scaling transformations method. By means of this method fractional order derivatives turns into integer order derivatives which allows us to deal with the easier problem. After establishing the solution and unknown coefficient of integer order diffusion problem, by utilizing the inverse transformation, we construct the solution and unknown coefficient of time fractional diffusion problem. Presented examples illustrate that identified unknown coefficient and the solution of the problem are in a high agreement with the exact solution of the corresponding the inverse problem.

\section{Introduction}

Last couple of decades fractional differential equations play a significant role in modelling of various processes. As a result, they attract growing attention of many scientists in diverse branches of sciences such as engineering, mathematics, chemistry and physics [1, 2, 3, 44, 5]. Consequently, numerous analytical and numerical methods have been utilized to construct

Received September 8, 2021.

AMS Subject Classification: 35R11.

Key words and phrases: Time fractional diffusion equation, fractional scaling transformations method, modified Riemann-Liouville fractional derivative, Inverse problem. 
solutions of mathematical problems including fractional differential equations [6, 7, 8, 9, $10,11,12$ ].

Therefore, identification of unknown coefficients in fractional differential equations with or without additional measured data becomes one of the trend challenges in inverse problems [13, 14, 15]. Hence, many researchers in various research areas have been developing new methods to tackle with this kind of inverse problems including fractional derivatives [13, 14, 15, 16, 17].

In this research, our focus is on establishing time dependent diffusivity coefficient and the solution of the mathematical problem including time fractional diffusion equation by means of fractional scaling transformation methods. The main advantage of this method is that it turns fractional order differential equations into integer order differential equations which makes the problem easier to tackle with. We remark that this method works out for the fractional differential equations in the sense of modified RiemannLiouville fractional derivative. The main goal in this article is to reveal the unknown coefficient of the following governing time fractional diffusion equation:

$$
D_{t}^{\alpha} u(x, t)=a(t) u_{x x}(x, t), 0<x<x_{1}, 0<t<t_{1}, 0<\alpha \leqslant 1,
$$

where $u(x, t)$ and $a(t)>0$ represent the temperature and thermal diffusivity, respectively. Associated to (1) the prescribed initial condition is

$$
u(x, 0)=\varphi(x), 0 \leqslant x \leqslant x_{1},
$$

and the prescribed Dirichlet boundary conditions are

$$
\begin{aligned}
u(0, t) & =\mu_{1}(t), 0<t \leqslant t_{1}, \\
u\left(x_{1}, t\right) & =\mu_{2}(t), 0<t \leqslant t_{1},
\end{aligned}
$$

with additional condition

$$
\int_{0}^{x_{1}} u(x, t) d x=E(t), 0<t \leqslant t_{1} .
$$

Having the condition $a(t)>0$ makes the problem (1)-(5) well-posed. 


\section{Preliminaries}

The modified Riemann-Liouville fractional calculus [18] is defined as

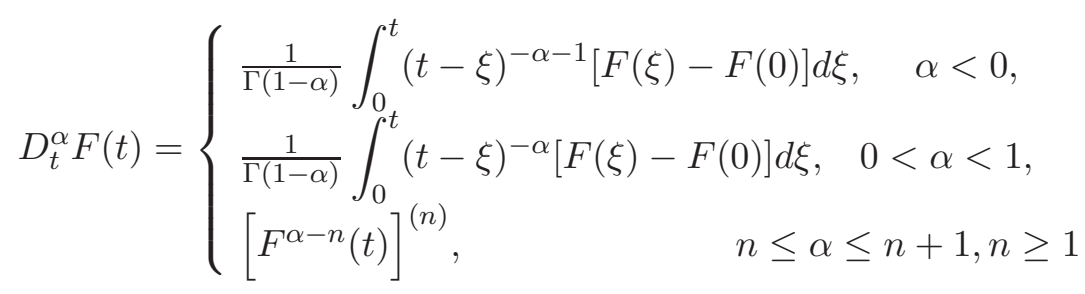

where $\alpha$ is a decimal, $\Gamma$ denotes the gamma function. According to the definitions of the fractional calculus Eq.(6), the following properties are held:

$$
\begin{aligned}
D_{t}^{\alpha} t^{\gamma} & =\frac{\Gamma(\gamma+1)}{\Gamma(\gamma-\alpha+1)} t^{\gamma-\alpha} \\
D_{t}^{\alpha}[F(t) G(t)] & =G(t)\left[D_{t}^{\alpha} F(t)\right]+F(t)\left[D_{t}^{\alpha} G(t)\right], \\
D_{t}^{\alpha} F[G(t)] & =F_{G}^{\prime}[G(t)] D_{t}^{\alpha} G(t)=\left(D_{G}^{\alpha} F[G(t)]\right)\left[G^{\prime}(t)\right]^{\alpha}
\end{aligned}
$$

where $F(t)$ and $G(t)$ are arbitrary functions, $\gamma>-1$ is a constant.

\section{Analysis of the New Fractional Derivative}

By means of the following fractional scaling transformations

$$
T=\frac{t^{\alpha}}{\Gamma(1+\alpha)}, u(x, t)=V(x, T),
$$

the problem (11)-(15) is converted to into the following integer order problem

$$
V_{T}=a(T) V_{x x}, 0<x<x_{1}, 0<T<\frac{t_{1}^{\alpha}}{\Gamma(1+\alpha)},
$$

with initial conditions

$$
V(x, 0)=\bar{\varphi}(x), 0<x \leqslant x_{1},
$$

and the prescribed Dirichlet boundary conditions are

$$
V(0, T)=\overline{\mu_{1}}(T), 0<T \leqslant \frac{t_{1}^{\alpha}}{\Gamma(1+\alpha)}
$$




$$
V\left(x_{1}, T\right)=\overline{\mu_{2}}(T), 0<T \leqslant \frac{t_{1}^{\alpha}}{\Gamma(1+\alpha)}
$$

and additional condition

$$
\int_{0}^{x_{1}} V(x, T) d x=\bar{E}(T), 0<T \leqslant \frac{t_{1}^{\alpha}}{\Gamma(1+\alpha)} .
$$

After establishing the solution and unknown coefficient of problem (11)-(15), by employing inverse transformation we obtain the solution $u(x, t)$ and an unknown diffusivity coefficient $a(t)$.

\section{Illustrative Examples}

In this section, we illustrate three examples of inverse problems about determination of unknown time dependent coefficient.

Example 1. Consider the inverse coefficient problem involving time fractional differential equations:

$$
\begin{aligned}
D_{t}^{\alpha} u(x, t) & =a(t) u_{x x}(x, t), \quad 0<x<1,0<t<(\Gamma(1+\alpha))^{\frac{1}{\alpha}}, \\
u(x, 0) & =\exp (x), \quad 0 \leqslant x \leqslant 1, \\
u(0, t) & =\exp \left(\frac{t^{\alpha}}{\Gamma(1+\alpha)}+\frac{t^{2 \alpha}}{(\Gamma(1+\alpha))^{2}}\right), \quad 0<t \leqslant(\Gamma(1+\alpha))^{\frac{1}{\alpha}} \\
u(1, t) & =\exp \left(1+\frac{t^{\alpha}}{\Gamma(1+\alpha)}+\frac{t^{2 \alpha}}{(\Gamma(1+\alpha))^{2}}\right), \quad 0<t \leqslant(\Gamma(1+\alpha))^{\frac{1}{\alpha}}, \\
\int_{0}^{1} u(x, t) d x=\exp \left(\frac{t^{\alpha}}{\Gamma(1+\alpha)}+\frac{t^{2 \alpha}}{(\Gamma(1+\alpha))^{2}}\right)(\exp (1)-1), & 0<t \leqslant(\Gamma(1+\alpha))^{\frac{1}{\alpha}} .
\end{aligned}
$$

By taking fractional scaling transformation methods into account the problem (16)-(20) turns into following integer order problem:

$$
V_{T}=a(T) V_{x x}, 0<x<1,0<T<1,
$$

with initial conditions

$$
V(x, 0)=\exp (x), 0<x \leqslant 1,
$$


and the prescribed Dirichlet boundary conditions are

$$
\begin{aligned}
& V(0, T)=\exp \left(T+T^{2}\right), 0<T \leqslant 1 \\
& V(1, T)=\exp \left(1+T+T^{2}\right), 0<T \leqslant 1
\end{aligned}
$$

and additional condition

$$
\int_{0}^{1} V(x, T) d x=\exp \left(T+T^{2}\right)(\exp (1)-1), 0<T \leqslant 1 .
$$

This inverse problem have the solution $V(x, T)=\exp \left(x+T+T^{2}\right)$ and unknown diffusivity coefficient becomes $a(T)=2 T+1$ [13]. As seen from Figs.1-4, by means of inverse transformation the solution of problem (21)(25) and unknown diffusivity coefficient are obtained in the following form respectively.

$$
u(x, t)=\exp \left(x+\frac{t^{\alpha}}{\Gamma(1+\alpha)}+\frac{t^{2 \alpha}}{(\Gamma(1+\alpha))^{2}}\right)
$$

and

$$
a(t)=b+c \frac{t^{\alpha}}{\Gamma(1+\alpha)} .
$$

where $b=1$ and $c=2$.

Moreover, the values of exact and approximate solutions of problem (16) $-(20)$ at $t=0.8$ for different values of orders of $\alpha$ are presented in Table 1 .

Table 1: The table of exact and approximate solution of Ex. 1 at $t=0.8$.

\begin{tabular}{|c|r|r|r|r|r|}
\hline$x$ & Exact & $\alpha=1$ & $\alpha=0.9$ & $\alpha=0.8$ & $\alpha=0.7$ \\
\hline 0 & 4.22070 & 4.22070 & 4.82614 & 5.50024 & 6.21906 \\
0.2 & 5.15517 & 5.15517 & 5.89466 & 6.71801 & 7.59598 \\
0.4 & 6.29654 & 6.29654 & 7.19976 & 8.20539 & 9.27775 \\
0.6 & 7.69061 & 7.69061 & 8.79381 & 10.02209 & 11.33186 \\
0.8 & 9.39333 & 9.39333 & 10.74078 & 12.24100 & 13.84077 \\
1 & 11.47304 & 11.47304 & 13.11882 & 14.95120 & 16.90516 \\
\hline
\end{tabular}




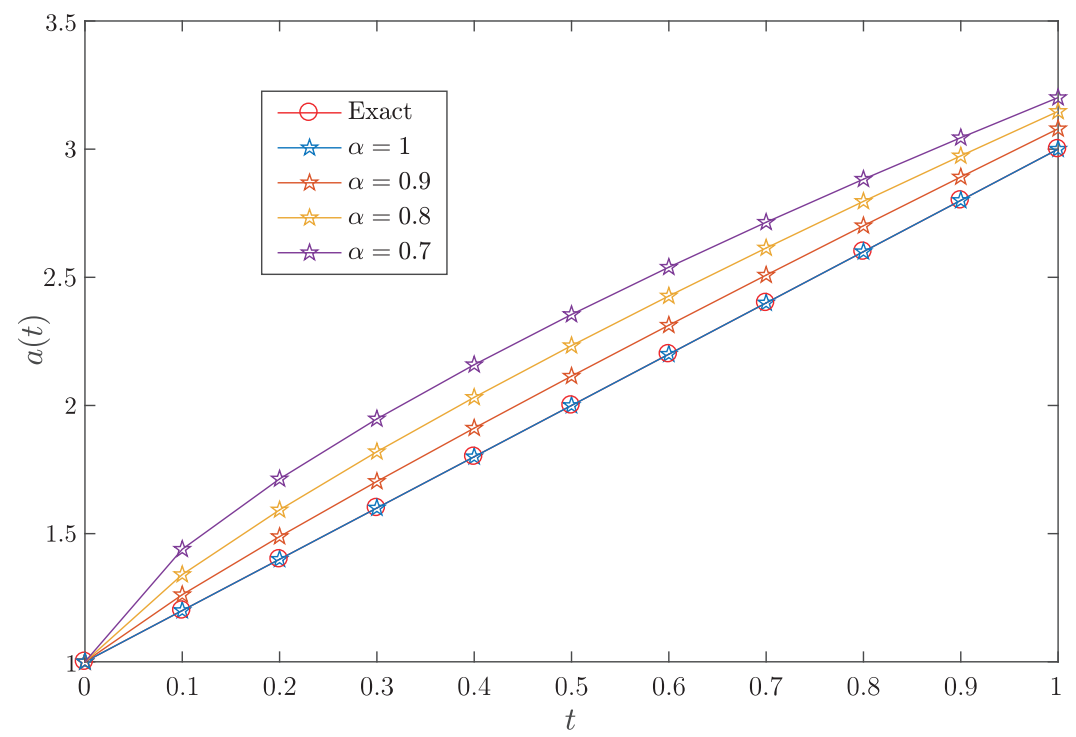

Figure 1: The graphics of approximate solution for $a(t)$ in Ex. 1 .

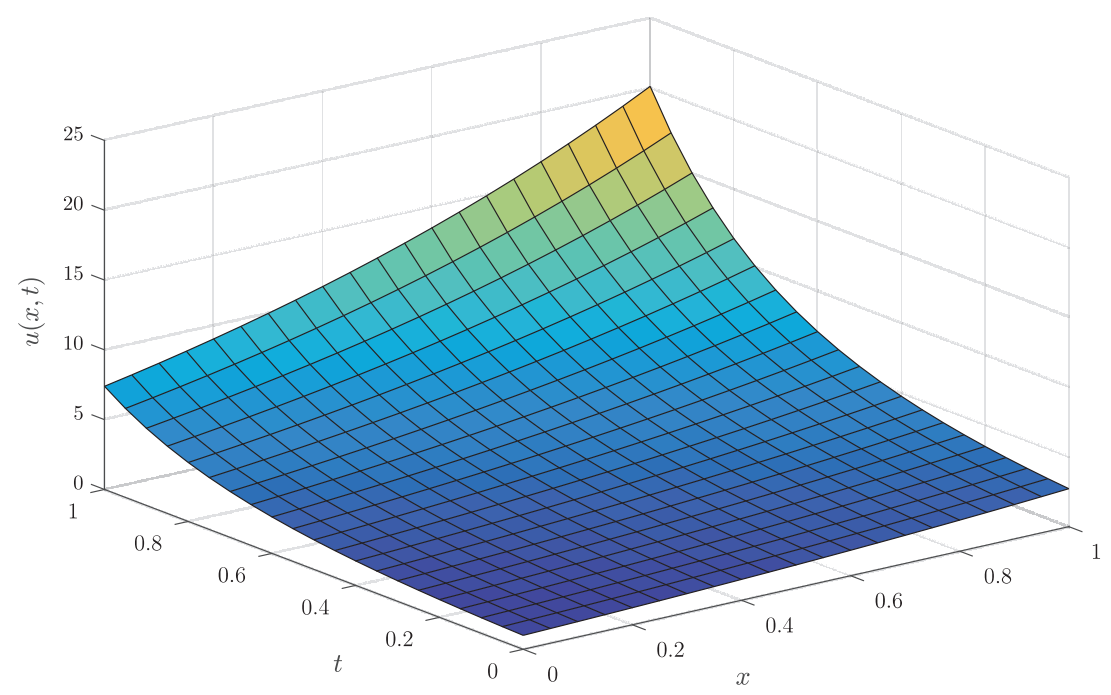

Figure 2: The graphics of exact solution for $u(x, t)$ in Ex. 1 . 


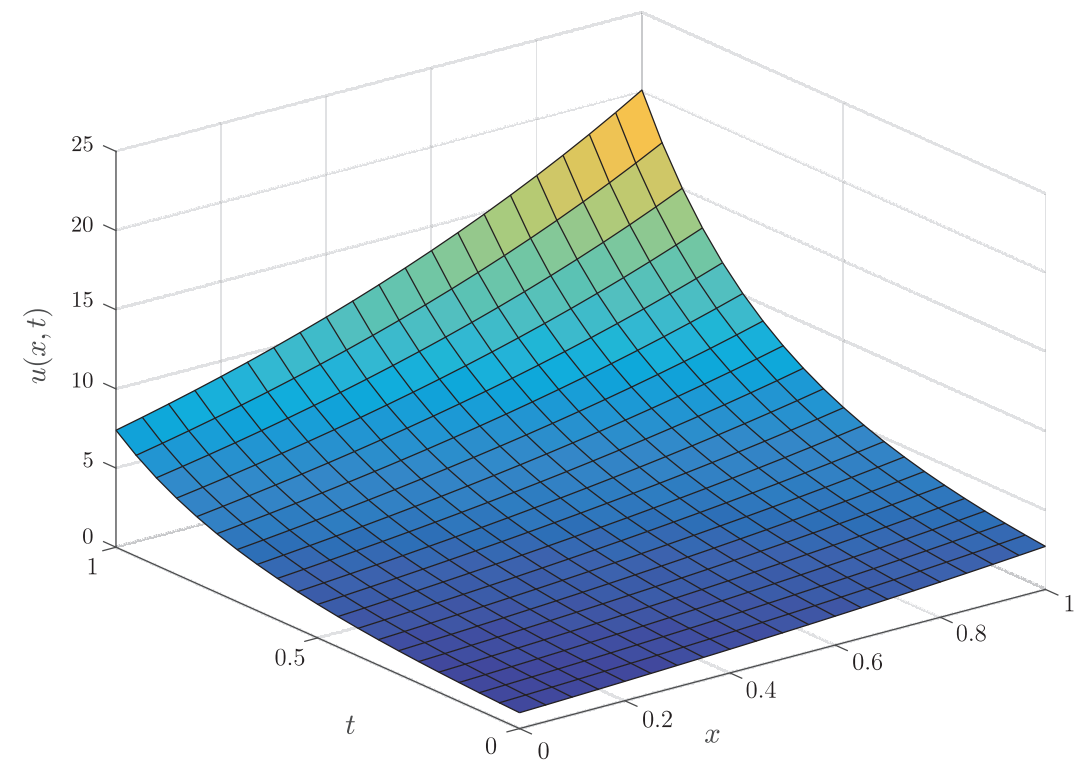

Figure 3: The graphics of approximate solution for $u(x, t)$ with $\alpha=1$ in Ex. 1 .

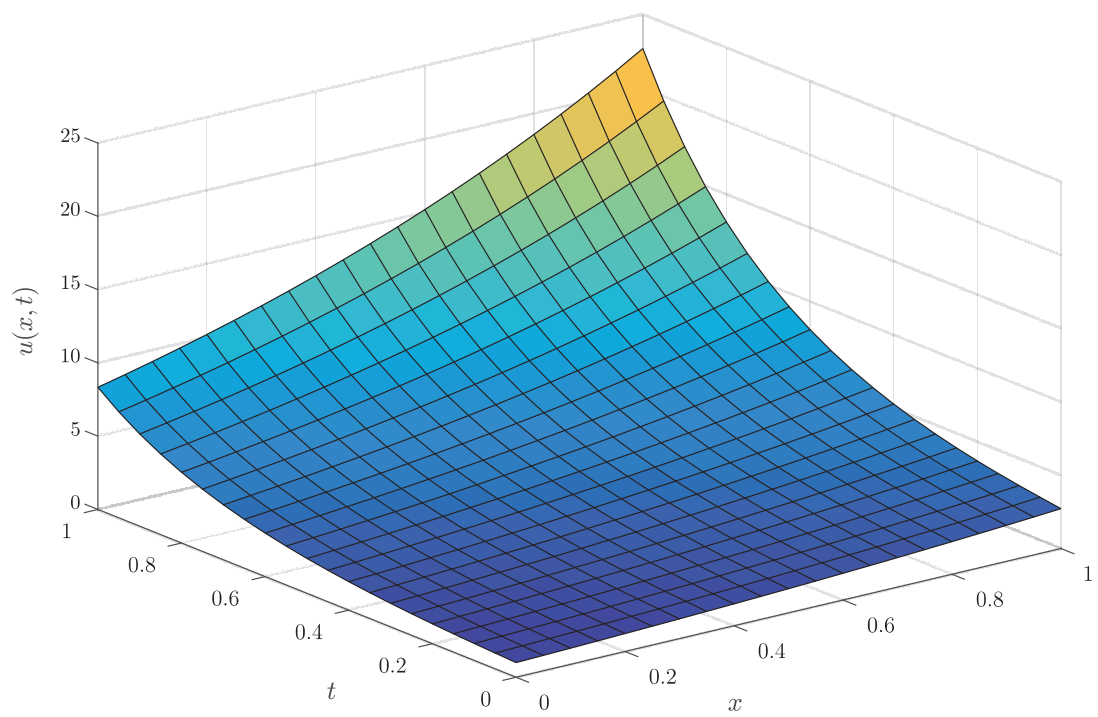

Figure 4: The graphics of approximate solution for $u(x, t)$ with $\alpha=0.9$ in Ex. 1 . 
Example 2. Consider the inverse coefficient problem involving time fractional differential equations:

$$
\begin{aligned}
& D_{t}^{\alpha} u(x, t)=a(t) u_{x x}(x, t), \quad 0<x<1,0<t<(\Gamma(1+\alpha))^{\frac{1}{\alpha}}, \\
& u(x, 0)=\exp (x), \quad 0 \leqslant x \leqslant 1, \\
& u(0, t)=\exp \left((4+\pi) \frac{t^{\alpha}}{\Gamma(1+\alpha)}-\frac{\pi^{3}}{6} \frac{t^{3 \alpha}}{(\Gamma(1+\alpha))^{3}}+\frac{\pi^{5}}{120} \frac{t^{5 \alpha}}{(\Gamma(1+\alpha))^{5}}\right), \\
& 0<t \leqslant(\Gamma(1+\alpha))^{\frac{1}{\alpha}} \\
& u(1, t)=\exp \left(1+(4+\pi) \frac{t^{\alpha}}{\Gamma(1+\alpha)}-\frac{\pi^{3}}{6} \frac{t^{3 \alpha}}{(\Gamma(1+\alpha))^{3}}+\frac{\pi^{5}}{120} \frac{t^{5 \alpha}}{(\Gamma(1+\alpha))^{5}}\right) \\
& 0<t \leqslant(\Gamma(1+\alpha))^{\frac{1}{\alpha}} \\
& \int_{0}^{1} u(x, t) d x=\exp \left((4+\pi) \frac{t^{\alpha}}{\Gamma(1+\alpha)}-\frac{\pi^{3}}{6} \frac{t^{3 \alpha}}{(\Gamma(1+\alpha))^{3}}\right. \\
& \left.+\frac{\pi^{5}}{120} \frac{t^{5 \alpha}}{(\Gamma(1+\alpha))^{5}}\right)(\exp (1)-1), \quad 0<t \leqslant(\Gamma(1+\alpha))^{\frac{1}{\alpha}} .
\end{aligned}
$$

By taking fractional scaling transformation methods into account the problem (28)-(32) turns into following integer order problem:

$$
V_{T}=a(T) V_{x x}, 0<x<1,0<T<1,
$$

with initial conditions

$$
V(x, 0)=\exp (x), 0<x \leqslant 1
$$

and the prescribed Dirichlet boundary conditions are

$$
\begin{aligned}
& V(0, T)=\exp \left((4+\pi) T-T^{3}+T^{5}\right), \quad 0<T \leqslant 1 \\
& V(1, T)=\exp \left(1+(4+\pi) T-T^{3}+T^{5}\right), \quad 0<T \leqslant 1
\end{aligned}
$$

and additional condition

$$
\int_{0}^{1} V(x, T) d X=\exp \left((4+\pi) T-T^{3}+T^{5}\right)(\exp (1)-1), 0<T \leqslant 1
$$

This inverse problem have the solution $V(x, T)=\exp \left(x+(4+\pi) T-T^{3}+T^{5}\right)$ and unknown diffusivity coefficient becomes $a(T)=(4+\pi)-\frac{\pi^{3} T^{2}}{2}+\frac{\pi^{5} T^{4}}{24}$ 
[13]. As seen from Figs.5-8, by means of inverse transformation the solution of problem (33)-(37) and unknown diffusivity coefficient are obtained in the following form respectively.

$$
u(x, t)=\exp \left(x+(4+\pi) \frac{t^{\alpha}}{\Gamma(1+\alpha)}-\frac{\pi^{3}}{6} \frac{t^{3 \alpha}}{(\Gamma(1+\alpha))^{3}}+\frac{\pi^{5}}{120} \frac{t^{5 \alpha}}{(\Gamma(1+\alpha))^{5}}\right)
$$

and

$$
a(t)=b+c \frac{t^{\alpha}}{\Gamma(1+\alpha)}+d \frac{t^{2 \alpha}}{(\Gamma(1+\alpha))^{2}}+e \frac{t^{3 \alpha}}{(\Gamma(1+\alpha))^{3}}+f \frac{t^{4 \alpha}}{(\Gamma(1+\alpha))^{4}}
$$

where $b=4+\pi, c=0, d=-\frac{\pi^{3}}{2}, e=0$ and $f=\frac{\pi^{5}}{24}$.

Moreover, the values of exact and approximate solutions of problem (16) $-(20)$ at $t=0.8$ for different values of orders of $\alpha$ are presented in Table 2 .

Table 2: The table of exact and approximate solution of Ex. 1 at $t=0.8$.

\begin{tabular}{|c|r|r|r|r|r|}
\hline$x$ & Exact & $\alpha=1$ & $\alpha=0.9$ & $\alpha=0.8$ & $\alpha=0.7$ \\
\hline 0 & 49.55301 & 49.55301 & 56.24642 & 64.10626 & 73.49413 \\
0.2 & 60.52418 & 60.52418 & 68.69953 & 78.29956 & 89.76593 \\
0.4 & 73.92440 & 73.92440 & 83.90980 & 95.63530 & 109.64036 \\
0.6 & 90.29146 & 90.29146 & 102.48766 & 116.80922 & 133.91504 \\
0.8 & 110.28224 & 110.28224 & 125.17871 & 142.67110 & 163.56420 \\
1 & 134.69904 & 134.69904 & 152.89362 & 174.25888 & 199.77776 \\
\hline
\end{tabular}




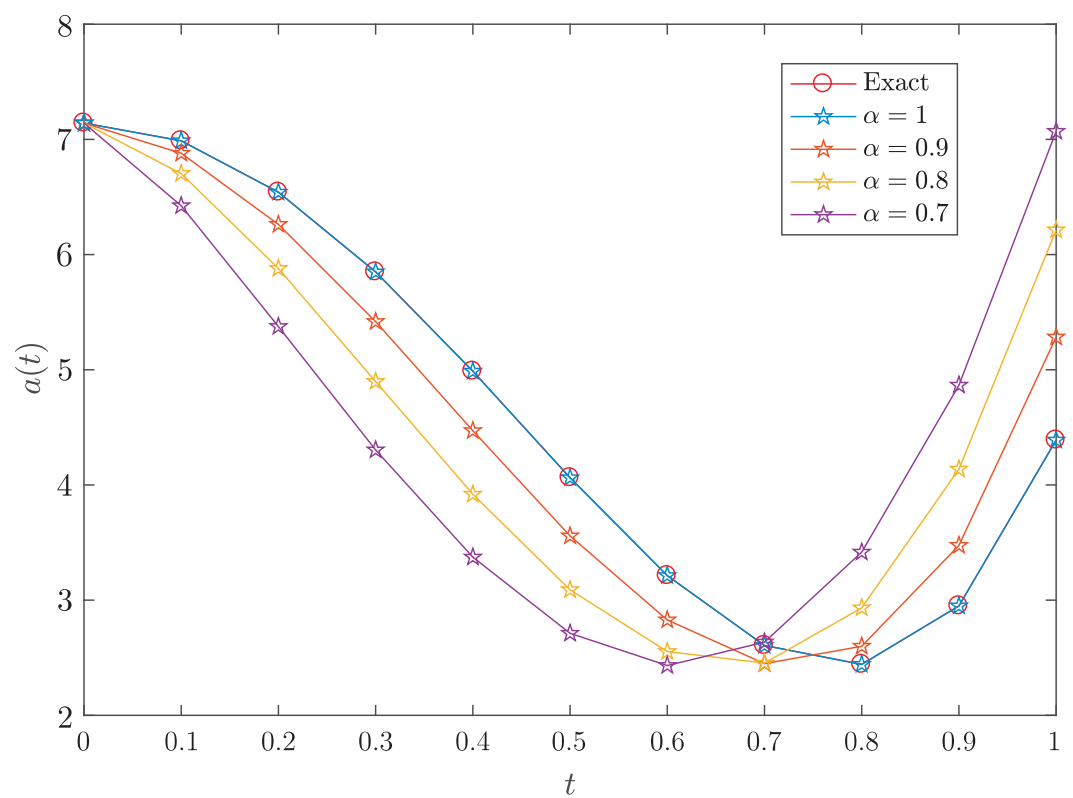

Figure 5: The graphics of approximate solution for $a(t)$ in Ex. 2 .

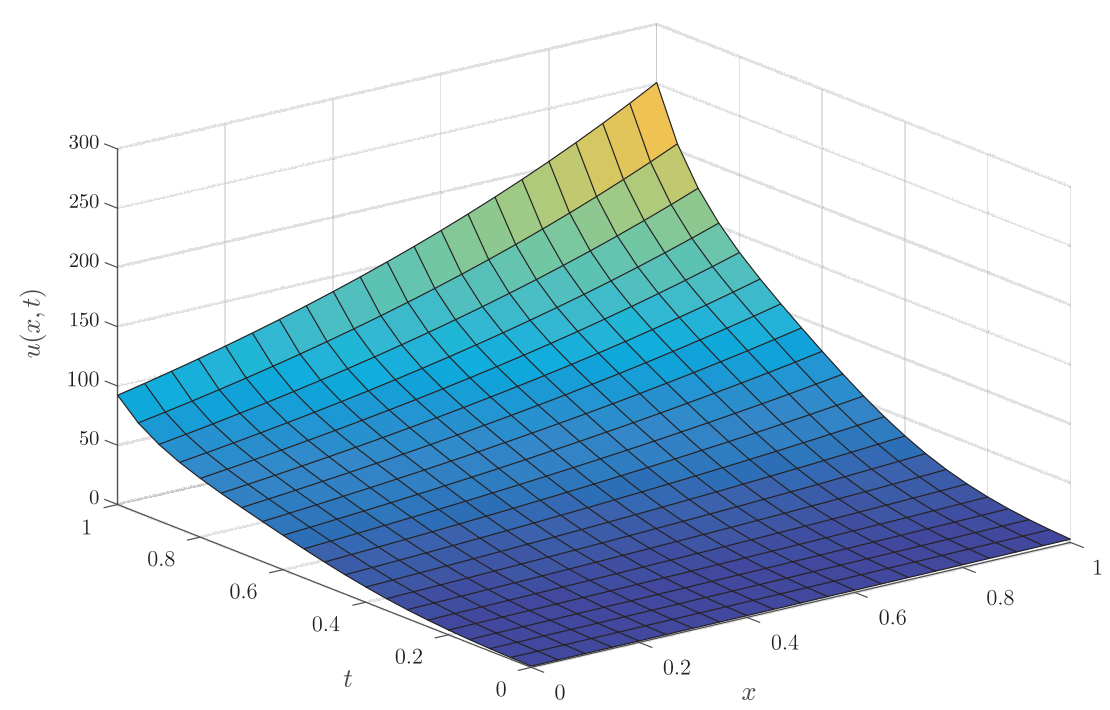

Figure 6: The graphics of exact solution for $u(x, t)$ in Ex. 2 . 


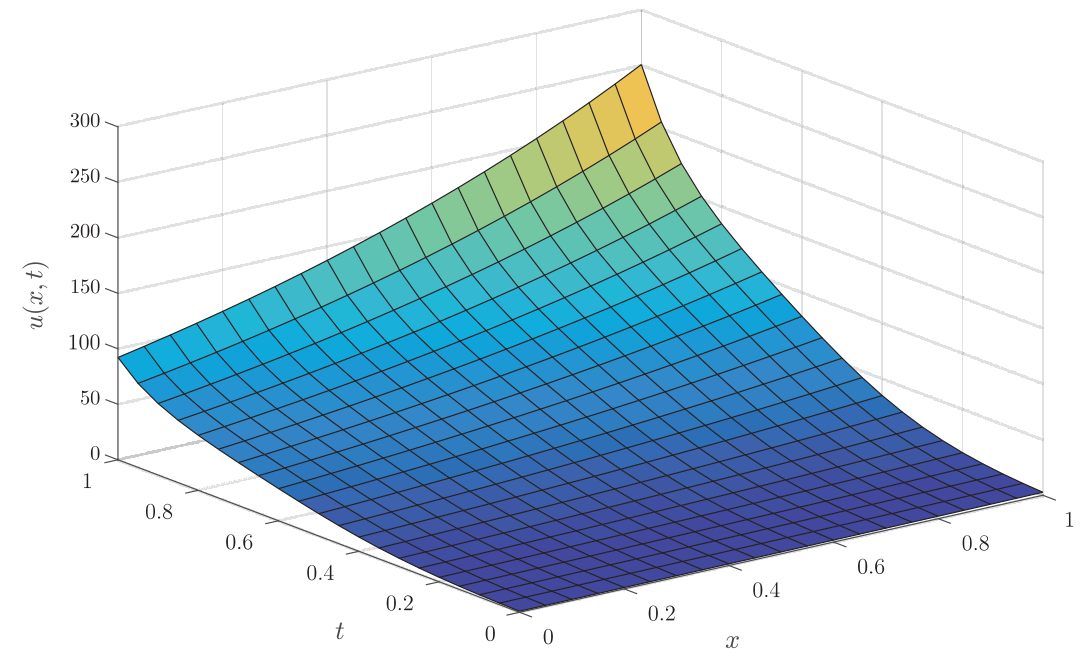

Figure 7: The graphics of approximate solution for $u(x, t)$ with $\alpha=1$ in Ex. 2 .

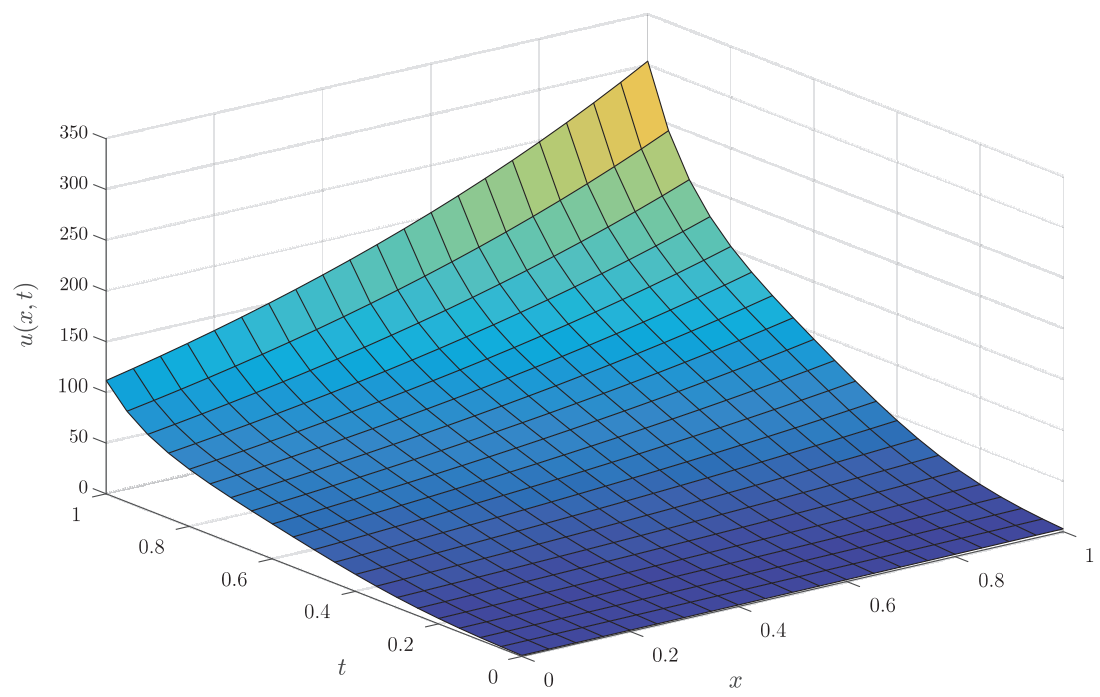

Figure 8: The graphics of approximate solution for $u(x, t)$ with $\alpha=0.9$ in Ex. 2 . 
Example 3. Consider the inverse coefficient problem involving time fractional differential equations:

$$
\begin{aligned}
& D_{t}^{\alpha} u(x, t)= a(t) u_{x x}(x, t), \quad 0<x<1,0<t<(\Gamma(1+\alpha))^{\frac{1}{\alpha}}, \\
& u(x, 0)= \begin{array}{l}
\exp \left(x+\frac{1}{4}\right), \quad 0 \leqslant x \leqslant \frac{1}{2}, \\
\exp \left(x+\frac{1}{2}\right), \quad \frac{1}{2} \leqslant x \leqslant 1,
\end{array} \\
& u(0, t)=\left\{\begin{array}{l}
\exp \left(\frac{1}{4}+\frac{t^{\alpha}}{\Gamma(1+\alpha)}-\frac{1}{2} \frac{t^{2 \alpha}}{(\Gamma(1+\alpha))^{2}}\right), 0 \leqslant t \leqslant\left(\frac{\Gamma(1+\alpha)}{2}\right)^{\frac{1}{\alpha}} \\
\exp \left(\frac{1}{2}+\frac{1}{2} \frac{t^{2 \alpha}}{(\Gamma(1+\alpha))^{2}}\right),\left(\frac{\Gamma(1+\alpha)}{2}\right)^{\frac{1}{\alpha}} \leqslant t \leqslant \Gamma(1+\alpha)^{\frac{1}{\alpha}},
\end{array}\right. \\
& \int_{0}^{1} u(1, t)=\left\{\begin{array}{l}
\exp \left(1+\frac{1}{4}+\frac{t^{\alpha}}{\Gamma(1+\alpha)}-\frac{1}{2} \frac{t^{2 \alpha}}{(\Gamma(1+\alpha))^{2}}\right), 0 \leqslant t \leqslant\left(\frac{\Gamma(1+\alpha)}{2}\right)^{\frac{1}{\alpha}}, \\
\exp \left(1+\frac{1}{2}+\frac{1}{2} \frac{t^{2 \alpha}}{(\Gamma(1+\alpha))^{2}}\right),\left(\frac{\Gamma(1+\alpha)}{2}\right)^{\frac{1}{\alpha}} \leqslant t \leqslant \Gamma(1+\alpha)^{\frac{1}{\alpha}},
\end{array}\right. \\
& \begin{array}{l}
\exp \left(\frac{1}{4}+\frac{t^{\alpha}}{\Gamma(1+\alpha)}-\frac{1}{2} \frac{t^{2 \alpha}}{(\Gamma(1+\alpha))^{2}}\right)(\exp (1)-1), 0 \leqslant t \leqslant\left(\frac{\Gamma(1+\alpha)}{2}\right)^{\frac{1}{\alpha}}, \\
\exp \left(\frac{1}{2}+\frac{1}{2} \frac{t^{2 \alpha}}{(\Gamma(1+\alpha))^{2}}\right)(\exp (1)-1),\left(\frac{\Gamma(1+\alpha)}{2}\right)^{\frac{1}{\alpha}} \leqslant t \leqslant \Gamma(1+\alpha)^{\frac{1}{\alpha}},
\end{array}
\end{aligned}
$$

By taking fractional scaling transformation methods into account the problem (40)-(44) turns into following integer order problem:

$$
V_{T}=a(T) V_{x x}, 0<x<1,0<T<1,
$$

with initial conditions

$$
V(x, 0)=\left\{\begin{array}{l}
\exp \left(x+\frac{1}{4}\right), 0 \leqslant x \leqslant \frac{1}{2} \\
\exp \left(x+\frac{1}{2}\right), \frac{1}{2} \leqslant x \leqslant 1
\end{array}\right.
$$

and the prescribed Dirichlet boundary conditions are

$$
\begin{gathered}
V(0, T)= \begin{cases}\exp \left(\frac{1}{4}+T-T^{2}\right), & 0 \leqslant T \leqslant \frac{1}{2}, \\
\exp \left(\frac{1}{2}+\frac{T^{2}}{2}\right), & \frac{1}{2} \leqslant T \leqslant 1,\end{cases} \\
V(1, T)= \begin{cases}\exp \left(\frac{5}{4}+T-T^{2}\right), & 0 \leqslant T \leqslant \frac{1}{2}, \\
\exp \left(\frac{3}{2}+\frac{T^{2}}{2}\right), & \frac{1}{2} \leqslant T \leqslant 1,\end{cases} \\
\int_{0}^{1} V(x, T) d x= \begin{cases}\exp \left(\frac{1}{4}+T-T^{2}\right)(\exp (1)-1), & 0 \leqslant T \leqslant \frac{1}{2}, \\
\exp \left(\frac{1}{2}+\frac{T^{2}}{2}\right)(\exp (1)-1), & \frac{1}{2} \leqslant T \leqslant 1 .\end{cases}
\end{gathered}
$$


This inverse problem have the solution

$$
V(x, T)= \begin{cases}\exp \left(x+\frac{1}{4}+T-T^{2}\right), & 0<x, T \leqslant \frac{1}{2}, \\ \exp \left(x+\frac{1}{2}+\frac{T^{2}}{2}\right), & \frac{1}{2} \leqslant x, T \leqslant 1\end{cases}
$$

and unknown diffusivity coefficient becomes

$$
a(T)= \begin{cases}1-T, & 0<T \leqslant \frac{1}{2} \\ T, & \frac{1}{2} \leqslant T \leqslant 1\end{cases}
$$

[13]. As seen from Figs.9-12, by means of inverse transformation the solution of problem (33)-(37) and unknown diffusivity coefficient are obtained in the following form respectively

$$
u(x, t)= \begin{cases}\exp \left(x+\frac{1}{4}+\frac{t^{\alpha}}{\Gamma(1+\alpha)}-\frac{1}{2} \frac{t^{2 \alpha}}{(\Gamma(1+\alpha))^{2}}\right), & 0 \leqslant x, t \leqslant \frac{1}{2}, \\ \exp \left(x+\frac{1}{2}+\frac{1}{2} \frac{t^{2 \alpha}}{(\Gamma(1+\alpha))^{2}}\right), & \frac{1}{2} \leqslant x, t \leqslant 1\end{cases}
$$

and

$$
a(t)=b+c \frac{t^{\alpha}}{\Gamma(1+\alpha)}
$$

where $b=1$ and $c=-1$ for $0 \leqslant t \leqslant \frac{1}{2}, b=0$ and $c=1$ for $\frac{1}{2} \leqslant t \leqslant 1$.

Moreover, the values of exact and approximate solutions of problem (16) $-(20)$ at $t=0.8$ for different values of orders of $\alpha$ are presented in Table 3 .

Table 3: The table of exact and approximate solution of Ex. 1 at $t=0.8$.

\begin{tabular}{|c|c|c|c|c|c|}
\hline$x$ & Exact & $\alpha=1$ & $\alpha=0.9$ & $\alpha=0.8$ & $\alpha=0.7$ \\
\hline 0 & 2.07508 & 2.07508 & 2.09350 & 2.10605 & 2.11337 \\
0.2 & 2.53451 & 2.53451 & 2.55700 & 2.57233 & 2.58127 \\
0.4 & 3.09566 & 3.09566 & 3.12313 & 3.14185 & 3.15277 \\
0.6 & 4.13712 & 4.13712 & 4.31345 & 4.49662 & 4.67913 \\
0.8 & 5.05309 & 5.05309 & 5.26846 & 5.49219 & 5.71510 \\
1 & 6.17186 & 6.17186 & 6.43491 & 6.70817 & 6.98044 \\
\hline
\end{tabular}




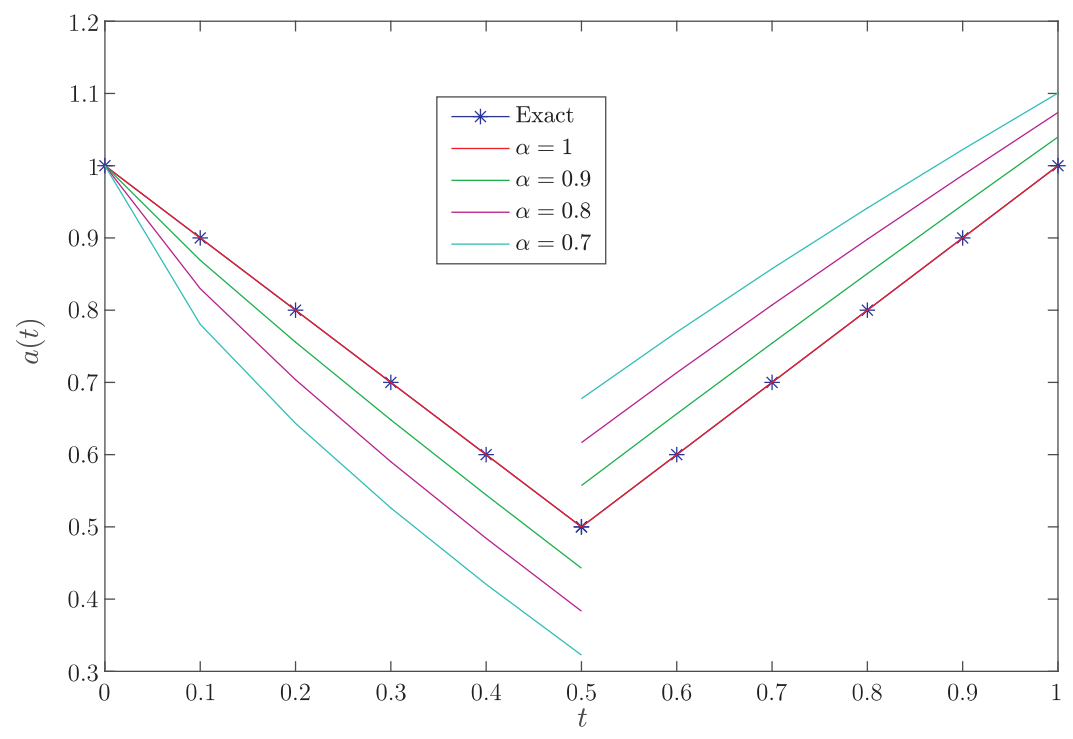

Figure 9: The graphics of approximate solution for $a(t)$ in Ex. 3 .

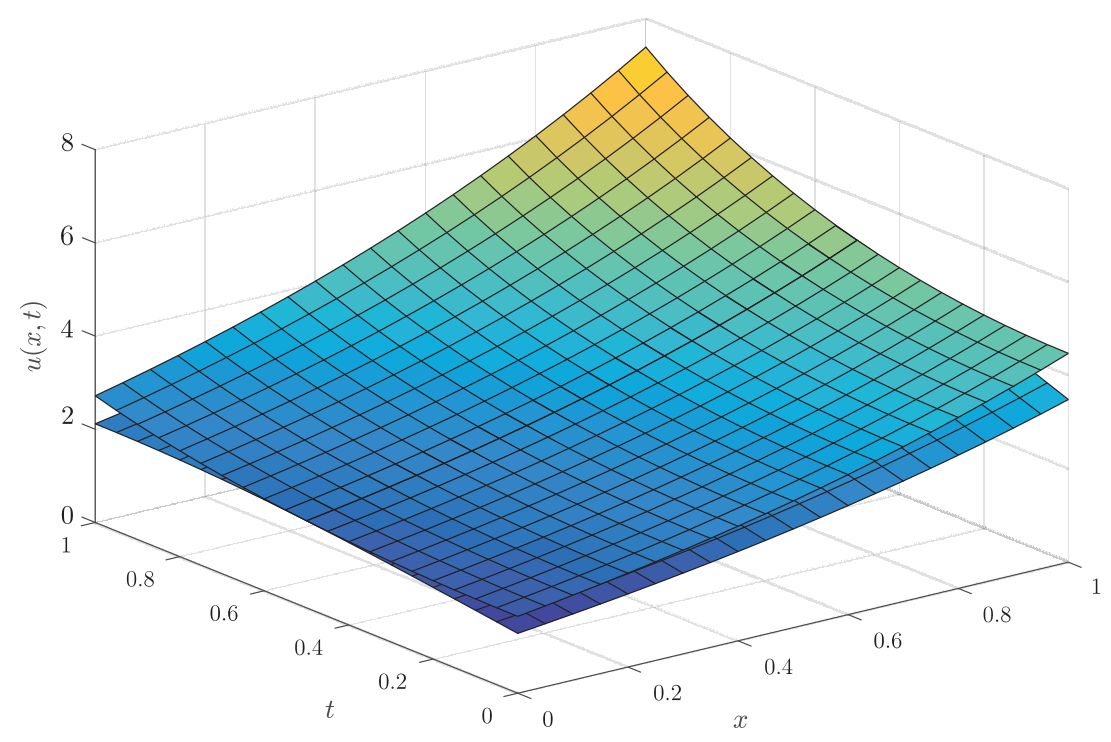

Figure 10: The graphics of exact solution for $u(x, t)$ in Ex. 3. 


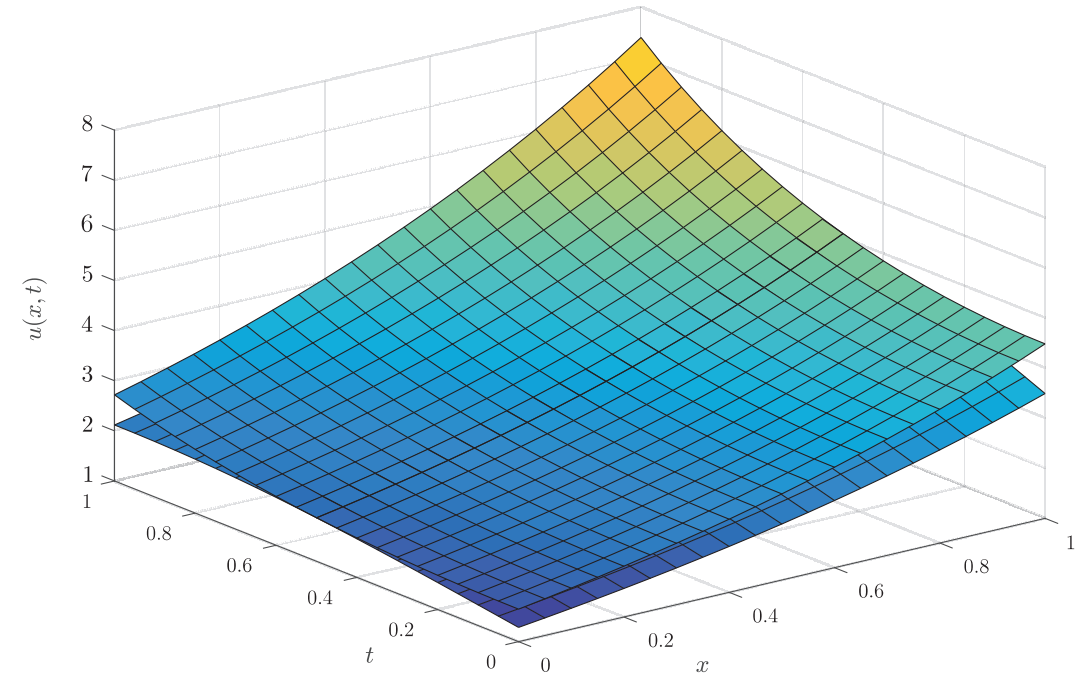

Figure 11: The graphics of approximate solution for $u(x, t)$ with $\alpha=1$ in Ex. 3 .

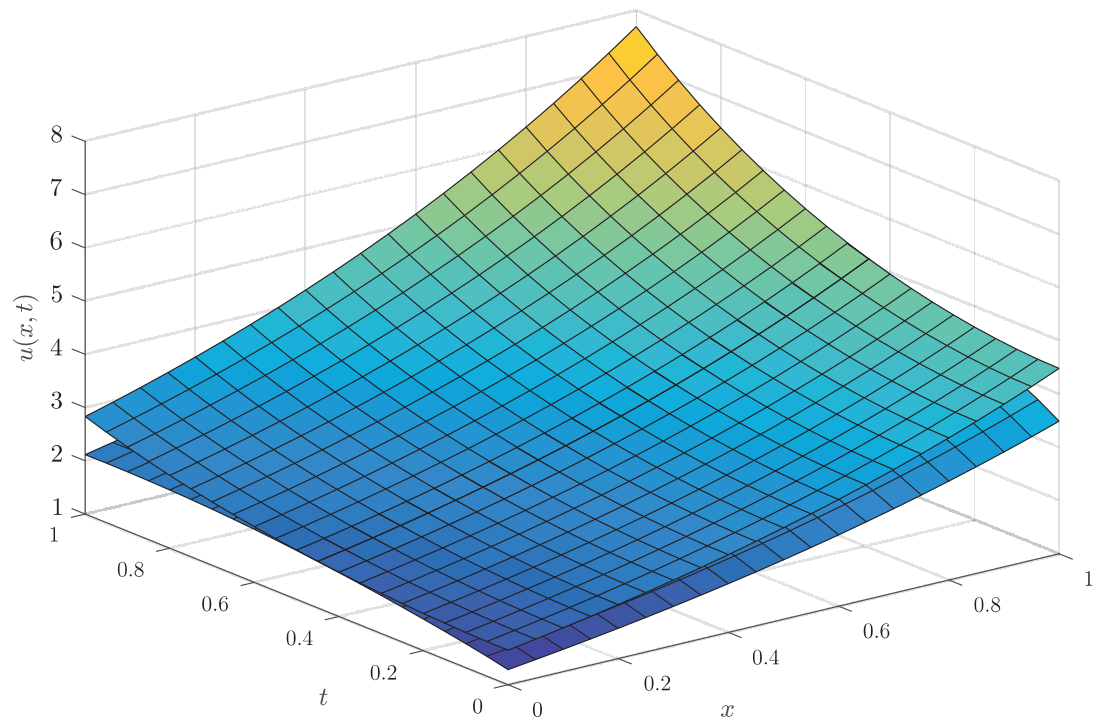

Figure 12: The graphics of approximate solution for $u(x, t)$ with $\alpha=0.9$ in Ex. 3 . 


\section{Conclusion}

In this research, the inverse problem of revealing unknown time-dependent diffusivity coefficient in mathematical problem including time fractional diffusion equation is taken into consideration. Fractional scaling transformation methods are implemented successfully to turn the fractional problem into integer order problem to determine the solution and to identify unknown time-dependent diffusion coefficient. The considerable advantage of this method is that we get rid of difficulties of fractional derivative which makes the problem easier to deal with. Future work will be concerned with the construction of the unknown parameter in time fractional differential equations with various boundary conditions.

\section{References}

1. K. B. Oldham, and J. Spanier, The Fractional Calculus: Theory and Applications of Differentiation and Integration to Arbitrary Order, Academic Press, 1974.

2. K. S. Miller, and B. Ross, An Introduction to the Fractional Calculus and Fractional Differential Equations, John Wiley and Sons, 1993.

3. L. A. Debnath, Recent applications of fractional calculus to science and engineering, Int. J. Math. Math. Sci., 54(2003), 3413-3442.

4. A. A. Kilbas, H. M. Srivastava, and J. J. Trujillo, Theory and Applications of Fractional Differential Equations, Elsevier, 2006.

5. I. Podlubny, Fractional Differential Equation, San Diego, CA: Academic Press, 1999.

6. J. Sabatier, O. P. Agarwal, and J. A. T. Machado, (eds), Advances in Fractional Calculus: Theoretical Developments and Applications in Physics and Engineering, Dordrecht: Springer, 2007.

7. S. G. Samko, A. A. Kilbas, and O. I. Marichev, Fractional Integrals and Derivatives Theory And Applications, Amsterdam: Gordon and Breach, 1993.

8. Z. Odibat, Approximations of fractional integrals and Caputo fractional derivatives, Appl. Math. Comput., 178(2006), 527-533.

9. S. Momani and Z. Odibat, Analytical approach to linear fractional partial differential equations arising in fluid mechanics, Phys. Lett. A, 355(2006), 271-279.

10. A. Atangana, On the new fractional derivative and application to nonlinear Fisherreaction- diffusion equation, Appl. Math. Comput., 273(2016), 948-956.

11. A. El-Ajou, O. Abu Arqub, S. Momani, D. Baleanu, and A. Alsaedi, A novel expansion iterative method for solving linear partial differential equations of fractional order, Appl. Math. Comput., 257(2015), 119-133. 
12. M. A. Bayrak and A. Demir, A new approach for space-time fractional partial differential equations by residual power series method, Appl. Math. Comput., 336(2018), $215-230$.

13. D. Lesnic, S. A. Yousefi and M. Ivanchov, Determination of a time-dependent diffusivity from nonlocal conditions, J. Appl. Math. Comput., 41(2013), 301-320.

14. X. Xiangtuan, G. Hongbo and Liu. Xiaohong, An inverse problem for a fractional diffusion equation, J. of Comput. Appl. Math., 236(2012), 4474-4484.

15. I. I. Mansur and C. Muhammed, Inverse source problem for a time-fractional diffusion equation with nonlocal boundary conditions, Appl. Math. Model., 40(2016), No.4, 891-899.

16. L. Songshu and F. Lixin, An Inverse Problem for a Two-Dimensional Time-Fractional Sideways Heat Equation, Math. Prob. Eng., Volume 2020, Article ID 5865971, 13 pages.

17. L. Zhiyuan, C. Xing, and L. Gongsheng, An inverse problem in time-fractional diffusion equations with nonlinear boundary condition, J. of Math. Phys., 60(2019), 091502.

18. G. Jumarie, Modified Riemann-Liouville derivative and fractional Taylor series of nondifferentiable functions further results, Comput. Math. Appl., 51(2006), 1367-1376. 